Article

\title{
Preparation and Hydrogen Storage Characteristics of Surfactant-Modified Graphene
}

\author{
Tao $\mathrm{Xu}{ }^{1}$, Jiayu Chen ${ }^{2}{ }^{\oplus}$, Wenhui Yuan ${ }^{3, *}$, Baoqing $\mathrm{Li}^{3}{ }^{3} \mathrm{Li} \mathrm{Li}^{4}$, Huijun $\mathrm{Wu}^{1}$ and Xiaoqing Zhou ${ }^{1}$ \\ 1 Academy of Building Energy Efficiency, School of Civil Engineering, Guangzhou University, \\ Guangzhou 510006, China; xutao9@mail.sysu.edu.cn (T.X.); wuhuijun@tsinghua.org.cn (H.W.); \\ zhou_xiaoqing03@163.com (X.Z.) \\ 2 Department of Architecture and Civil Engineering, City University of Hong Kong, Tat Chee Avenue, \\ Kowloon, Hong Kong, China; jiaychen@cityu.edu.hk \\ 3 Key Laboratory of Enhanced Heat Transfer and Energy Conservation, The Ministry of Education, \\ School of Chemistry and Chemical Engineering, South China University of Technology, \\ Guangzhou 510640, China; lbqscut@163.com \\ 4 School of Environment Energy, South China University of Technology, Guangzhou 510006, China; \\ lli@scut.edu.cn \\ * Correspondence: cewhyuan@scut.edu.cn; Tel.: +86-020-8711-1887
}

Received: 19 October 2018; Accepted: 31 October 2018; Published: 2 November 2018

\begin{abstract}
As the depletion of traditional fossil fuels and environmental pollution become serious problems for human society, researchers are actively looking for renewable energy sources. Since hydrogen energy is considered a clean, efficient, and renewable alternative energy source, it is regarded as the most promising option. In this context, how to store hydrogen safely and efficiently has become the major challenge that hinders the actual application. To fill this gap, this paper proposes to utilize surfactant-modified graphene for hydrogen storage. Through a modified Hummers' method and ultrasonic stripping, this study proposes to prepare graphene from graphite oxide with $\mathrm{NaBH}_{4}$. The surfactant sodium dodecyl benzene sulfonate (SDBS) was used as a dispersant during the reduction process to produce dispersion-stabilized graphene suspensions. Then, to investigate the characteristics of the graphene suspensions, $\mathrm{X}$-ray diffraction (XRD), SEM, TEM, Fourier transform infrared (FT-IR), Raman, XPS, TG, and $\mathrm{N}_{2}$ adsorption-desorption tests were conducted. Finally, analytical models for hydrogen adsorption were investigated with Langmuir and Freundlich fittings. The results show that the application of SDBS can effectively reduce the agglomeration among graphene monolayers and increase the specific surface area of graphene, and that the adsorption behavior is consistent with the Freundlich adsorption model, and is a physical process.
\end{abstract}

Keywords: surfactant; graphene; hydrogen storage; adsorption

\section{Introduction and Background}

Energy and the environment are two major issues that mankind needs to confront. With the depletion of traditional fossil fuels and serious environmental pollution, researchers have come to an agreement on the necessity of alternative clean and renewable sources. Hydrogen energy is recognized as one of the ideal options for its cleanness, efficiency, and renewability [1-6]. The energy density obtained from hydrogen is almost three times higher than that of gasoline, and about seven times higher than that of coal in an equal mass [7]. However, hydrogen is unstable, and in order to safely and efficiently store hydrogen is a crucial barrier impeding its wide application. Proper hydrogen storage should involve high energy density, low energy waste, and high reliability. For example, the renewable energy generating systems in buildings rely on hydrogen to store the produced energy for commercial buildings [8,9] and infrastructures [10]. 
Researchers usually use mass storage density and volume storage density as two major indicators for hydrogen storage. Many researchers have carried out extensive and profound studies on hydrogen storage technologies in past decades [11,12]. One branch of study involves the storage materials, which categorizes hydrogen storage as physical adsorption and chemisorption based on material types. Good hydrogen storage materials should allow rapid storage and a high volumetric and gravimetric density of hydrogen. There are four main types of adsorption materials, including carbon hydrogen storage materials, non-carbon nanotube materials [13], mineral porous materials, and microporous metal-organic frameworks [14]. The carbon hydrogen storage materials are more popular, as they are insensitive to a small amount of gaseous impurities, and can be used repeatedly. In addition, they have low atomic mass, high chemical stability, and rich tubing structures [15-20]. Carbon hydrogen storage materials also have subcategories, including super activated carbon, nanostructured carbon materials, and graphene [21]. Graphene is considered as the prospective new carbonaceous hydrogen storage material with the highest potential due to its unique physical and chemical properties and simplicity of preparation. When modified by metals such as $\mathrm{Pd}, \mathrm{Pt}, \mathrm{Ni}, \mathrm{Ti}, \mathrm{Sc}, \mathrm{V}, \mathrm{Cu}$, etc., graphene can further improve its hydrogen storage capacity [22-24].

The preparation of graphene has made great breakthroughs in past decades. Recent studies have mainly focused on increasing the specific surface area of graphene materials. Srinivas et al. oxidized graphite with a modified Hummers' method and reduced hydrated hydrazine to produce graphene [25]. Under a pressure of $1000 \mathrm{kPa}$ and temperatures of $-196^{\circ} \mathrm{C}$ and $25^{\circ} \mathrm{C}$, the absorbed amount of hydrogen can reach $1.2 \mathrm{wt} \%$ and $0.1 \mathrm{wt} \%$, respectively. Ghosh et al. produced graphene through the thermal exfoliation of graphite oxide at high temperature [26]. Their results show that the hydrogen adsorption capacity was about $2.0-3.1 \mathrm{wt} \%$ at $10,000 \mathrm{kPa}$ and $65^{\circ} \mathrm{C}$. Cheng et al. reported that the hydrogen adsorption capacity of graphene reaches $0.4 \mathrm{wt} \%$ and $0.2 \mathrm{wt} \%$ at $100 \mathrm{kPa},-196{ }^{\circ} \mathrm{C}, 6000 \mathrm{kPa}$, and $25^{\circ} \mathrm{C}$, respectively [27]. In addition, various graphene preparation studies show that, due to agglomeration, the actual specific surface area of the produced graphene is much smaller than its theoretical value $(6.0 \mathrm{wt} \%)$. Therefore, this study aims to obtain a stable graphene suspension and prepare high-quality single-layer graphene in order to improve hydrogen adsorption.

Graphene has low hydrophilicity and lipophilicity, which tends to cause agglomeration. Without preprocessing, it is difficult to obtain monolayers and aqueous solutions or organic reagents. The dispersion of graphene may form lumps and agglomerates, which further deteriorate its performance after drying. Therefore, it is necessary to improve the hydrophilicity or lipophilicity of graphene so that the formation of composites and other substances can be reduced. In recent years, researchers found that the surface of graphite oxide contains many active groups, such as carbonyl groups, carboxyl groups, epoxy groups, etc. These groups can react with other substances, making the modification of graphene feasible, and thus leading to an active graphene surface. In general, the modification methods for graphene mainly include surface modification, chemical doping, functionalization of a polymer base, and physical modification. Bourlino et al. utilized amino siloxane and amino acid for its modification, and the modified graphene can remain stable in water [28]. Niyogi et al. selected octadecylamine (ODA) as a modifier to synthesize long-chain alkylated graphene on the surface of graphite oxide [29]. The modified graphene can disperse in tetrahydrofuran and carbon tetrachloride solutions. Ensafi et al. used a spillover mechanism to improve the hydrogen storage capacity of layered double hydroxides/reduced graphene, and the presence of $o$-phenylenediamine and $p$-phenylenediamine improved the kinetics of the hydrogen adsorption [30]. Zhao et al. used the graphene sulfonation method, which can not only improve the graphene, but also maintain the properties of graphene well [31]. This method first uses the sodium borohydride to allow a reduction-oxidation reaction, and then, the product was sulfonated in an ice bath for $2 \mathrm{~h}$. Finally, bisamine was used as a reducing agent for chemical reduction. Liang et al. used charge repulsion to obtain a well-dispersed graphene suspension in water [32]. Niyogi et al. reported that octadecylamine can be used to treat long-chain alkyl modified graphene [29]. Based upon the 
aforementioned studies, this paper proposes a new method to prepare high-quality graphene for the purpose of hydrogen storage.

\section{Methodology}

\subsection{Materials and Apparatus}

The experiment materials and equipment are summarized in following Tables 1 and 2.

Table 1. Chemicals used in the experiment. SDBS: sodium dodecyl benzene sulfonate.

\begin{tabular}{ccccc}
\hline Name & Chemical Formula & Molecular Weight & Level & Producer \\
\hline Plake Graphite & $\mathrm{C}$ & 12.01 & 325 mesh, 99.8\% & $\begin{array}{c}\text { Alfa Aesar Tianjin Chemical Co., Ltd., } \\
\text { Tianjin, China }\end{array}$ \\
\hline Ptassium Permanganate & $\mathrm{KMnO}_{4}$ & 158.04 & Analytically Pure & $\begin{array}{c}\text { China Sinopharm Chemical Reagent Co., } \\
\text { Ltd., Shanghai, China }\end{array}$ \\
\hline Hydrogen Peroxide & $\mathrm{H}_{2} \mathrm{SO}_{4}$ & 98.01 & $95.0-98.0 \%$ & $\begin{array}{c}\text { China Sinopharm Chemical Reagent Co., } \\
\text { Ltd., Shanghai, China }\end{array}$ \\
\hline Potassium Perchlorate & $\mathrm{H}_{2} \mathrm{O}_{2}$ & 34.01 & $30 \%$ & $\begin{array}{c}\text { China Sinopharm Chemical Reagent Co., } \\
\text { Ltd., Shanghai, China }\end{array}$ \\
\hline Muriatic Acid & $\mathrm{KClO}_{4}$ & 138.56 & Analytically Pure & $\begin{array}{c}\text { China Sinopharm Chemical Reagent Co., } \\
\text { Ltd., Shanghai, China }\end{array}$ \\
\hline Sodium Borohydride & $\mathrm{HCl}$ & 36.56 & $36-38 \%$ & $\begin{array}{c}\text { China Sinopharm Chemical Reagent Co., } \\
\text { Ltd., Shanghai, China }\end{array}$ \\
\hline Sodium Nitrate & $\mathrm{NaBH}_{4}$ & 37.85 & Analytically Pure & $\begin{array}{c}\text { China Sinopharm Chemical Reagent Co., } \\
\text { Ltd., Shanghai, China }\end{array}$ \\
\hline Acetone & $\mathrm{NaNO}_{3}$ & 84.99 & Analytically Pure & $\begin{array}{c}\text { China Sinopharm Chemical Reagent Co., } \\
\text { Ltd., Shanghai, China }\end{array}$ \\
\hline SDBS & $\mathrm{CH}_{3} \mathrm{COCH}_{3}$ & 58.08 & Analytically Pure & $\begin{array}{c}\text { China Sinopharm Chemical Reagent Co., } \\
\text { Ltd., Shanghai, China }\end{array}$ \\
\hline $\mathrm{C}_{18} \mathrm{H}_{29} \mathrm{NaO}_{3} \mathrm{~S}$ & 348.48 & Analytically Pure & $\begin{array}{c}\text { China Sinopharm Chemical Reagent Co., } \\
\text { Ltd., Shanghai, China }\end{array}$ \\
\hline
\end{tabular}

Table 2. List of apparatus.

\begin{tabular}{cc}
\hline Equipment & Producer \\
\hline Sartorius Electronic Scales & Beijing Sartorius Instrumentation System Co., Ltd., Beijing, China \\
DHG-9070A Electro-thermostatic Blast Oven & China Shanghai Yiheng Science and Technology Ltd., Shanghai, China \\
SHZ-D Circulating Vacuum Pump & China Gongyi Yuhua Instrumentation Co., Ltd., Gongyi, Henan, China \\
KQ-500VDF Ultrasonic Generator & China Kunshan Ultrasound Instrumentation Co., Ltd., Suzhou, Jiangsu, China \\
IKL RCT Basic Magnetic Stirrers & China Shanghai Yiheng Science and Technology Ltd., Shanghai, China \\
HC-3518 Centrifuge & China USTC Chuangxin Co., Ltd., Hefei, Anhui, China \\
\hline
\end{tabular}

\subsection{Preparation of Surfactant Modified Graphene}

\subsubsection{Preparation of Graphite Oxide}

A modified Hummers' method was used to prepare graphite oxide. The experimental method is shown in Figure 1. First, a 500-mL reaction flask was assembled in an ice water bath. Then, $5 \mathrm{~g}$ of graphite powder and $5 \mathrm{~g}$ of sodium nitrate with $200 \mathrm{~mL}$ of concentrated sulfuric acid were added in the flask. Under stirring, $25 \mathrm{~g}$ of potassium perchlorate and $15 \mathrm{~g}$ of permanganate were introduced. The reaction temperature was kept below $20{ }^{\circ} \mathrm{C}$. If the reactions finished, the reaction bottle was removed from the ice bath and stirred on an electromagnetic stirrer for $24 \mathrm{~h}$. After that, $200 \mathrm{~mL}$ of deionized water was added slowly, and the temperature was raised to about $98^{\circ} \mathrm{C}$. After stirring for $20 \mathrm{~min}$, an appropriate amount of hydrogen peroxide was added to reduce the remaining oxidizing agent until the solution became bright yellow. The graphite oxide suspension was then centrifuged at a rate of 10,000 $\mathrm{rpm}$ and washed successively with $5 \% \mathrm{HCl}$ solution and deionized water until the $\mathrm{pH}$ of the separation solution reached 7.0. The obtained cake was vacuum dried to obtain graphite oxide (GO). 


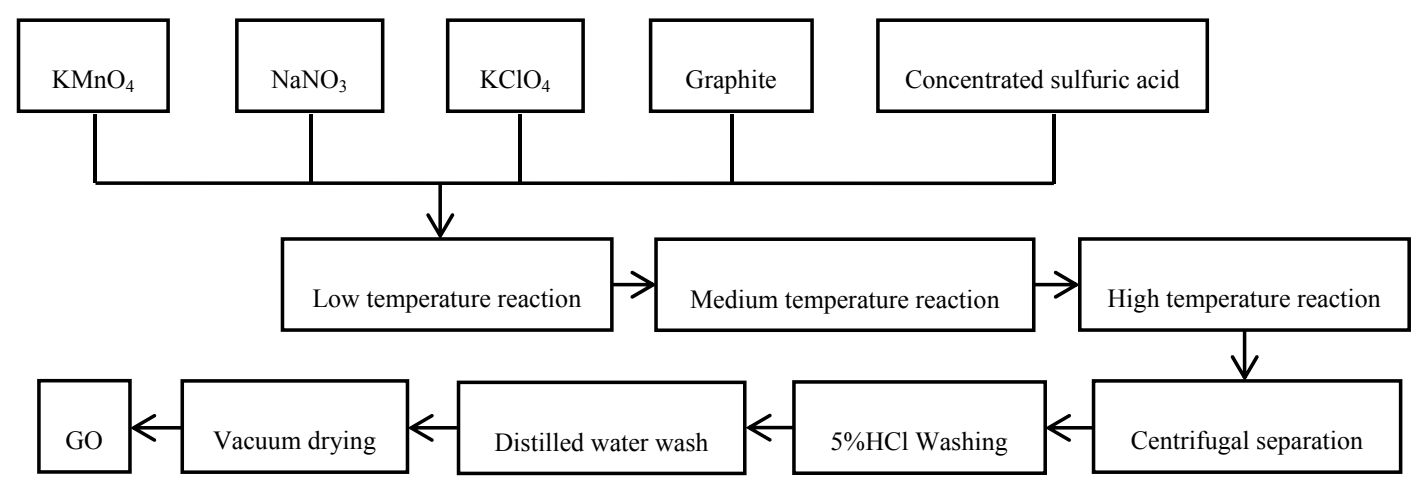

Figure 1. Flow chart of preparation of graphite oxide.

\subsubsection{Preparation of Graphene}

Figure 2 shows the method of preparation of graphene by sodium dodecyl benzene sulfonate (SDBS). First, the pulverization of GO was carried out via the spray-drying method. Then, $0.25 \mathrm{~g} / \mathrm{L} \mathrm{GO}$ aqueous solution was pulverized on a spray dryer at $200{ }^{\circ} \mathrm{C}$ with a flow rate of $300 \mathrm{~mL} / \mathrm{h}$, and a gas flow rate of $0.7 \mathrm{~m}^{3} / \mathrm{h}$. After the pulverization, $300 \mathrm{mg}$ of graphite oxide was dispersed in $60 \mathrm{~mL}$ of deionized water to obtain a brownish-yellow suspension. A stable colloidal suspension can be obtained after ultrasonic dispersion for $1 \mathrm{~h}$. Then, the suspension was transferred to a four-necked flask, and $600 \mathrm{mg}$ of sodium borohydride and $50 \mathrm{mg}$ of sodium dodecyl benzene sulfonate (SDBS) were added at $80^{\circ} \mathrm{C}$. After refluxing for $16 \mathrm{~h}$, the mixture was centrifuged and washed with acetone and deionized water until a $\mathrm{pH}$ of 7 was reached. The obtained filter cake was vacuum dried and stored for future use. This product was denoted as GS1. The other graphene that was prepared without a dispersing agent was denoted as GS.

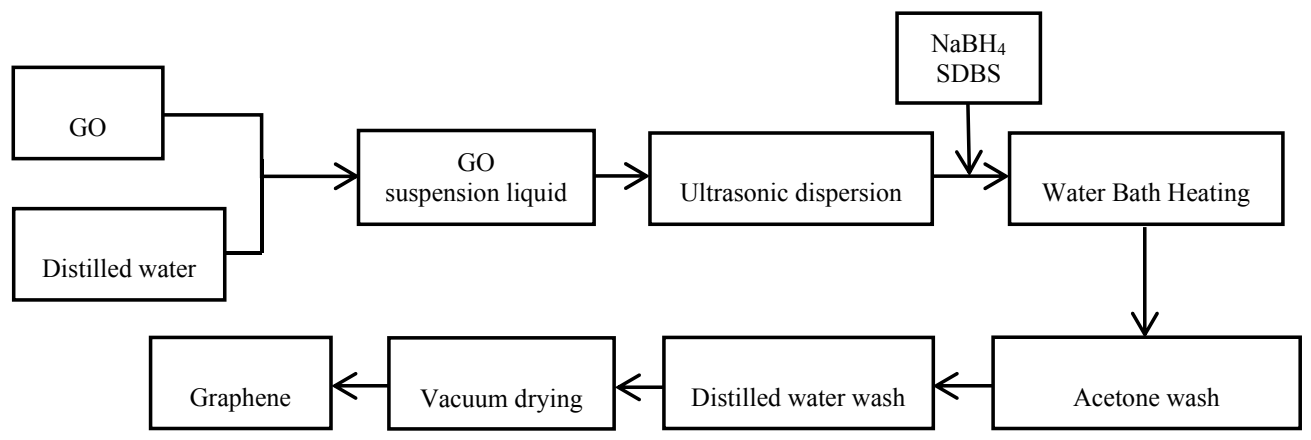

Figure 2. Flow chart of preparation of graphene by SDBS.

\subsubsection{Inspection of Physical Structure}

An X-ray diffraction (XRD) test was conducted with a D8 polycrystalline powder diffractometer (Bruker, Ettlingen, Germany) to analyze the crystal structure of the prepared graphite oxide and graphene samples. In the test, with $\mathrm{Cu}$ Ka radiation, the $\lambda=1.54 \times 10^{-10} \mathrm{~m}$ line was used as the ray source with a tube flow of $30 \mathrm{~mA}$ (tube pressure is $40 \mathrm{kV}$, and the scanning range is $5^{\circ}-60^{\circ}$ ). The infrared absorption spectrum of the samples was measured with a Vector 33 Fourier Transform Infrared Spectrometer (Bruker, Germany) with a scanning range of $4000-400 \mathrm{~cm}^{-1}$. KBr tablets were used for sample preparation. Then, the samples were tested with a Raman spectrometer (LabRAM Aramis, HJY, Paris, France) at room temperature. The excitation line was a 632.8-nm line of an Ar ion laser. One measurement included 20 scans with $20 \mathrm{~mW}$ of power. The X-ray

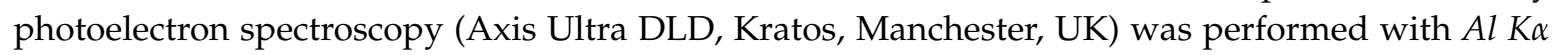
radiation $(15 \mathrm{kV}, 10 \mathrm{~mA}, \mathrm{hv}=1486.6 \mathrm{eV})$ as the excitation source. The water content and composition of samples were examined by a TG analyzer (SAT449C, NETZSCH, Bavaria, Germany). The test was carried out under an atmosphere of oxygen (gas flow: $30 \mathrm{~mL} / \mathrm{min}$ ) and nitrogen (gas flow: 
$28 \mathrm{~mL} / \mathrm{min}$ ), and a temperature between $20-800{ }^{\circ} \mathrm{C}$ with a heating rate of $5{ }^{\circ} \mathrm{C} / \mathrm{min}$. The TG test aims to verify whether the sample was sufficiently dry and the graphite oxide was completely reduced. The morphology and roughness of the sample surface were inspected with an atomic force microscope (Veeco Multimode 3D, Veeco, NJ, USA). For the inspection, $1 \mathrm{mg} / \mathrm{L}$ graphene suspensions with water dispersion medium were prepared. The surface morphology and structure of graphene were also inspected with a scanning electron microscope (S-3700N, Hitachi, Tokyo, Japan). Gold films were sprayed on the surface of the sample with $5 \mathrm{~min}$ of gold plating time. The ultrastructure of the graphene surface was observed with a transmission electron microscope (Tecnai G2 F30 S-Twin, Philips-FEI, Amsterdam, The Netherlands). The measurement of an $\mathrm{N}_{2}$ adsorption-desorption isotherm was studied with a specific surface area analyzer (ASAP 2010, Micromeritics, Atlanta, GA, USA), which had liquid nitrogen as a dispersion medium $(77 \mathrm{~K})$. According to the characteristics of the graphene sample $\mathrm{N}_{2}$ absorption capacity, the surface area of the sample was calculated based on the Brunauer-Emmett-Teller (BET) equation [33]. The pore size distribution curve was calculated by the Barrett-Joyner-Halenda (BHJ) method with desorption branches [34].

\subsubsection{Inspection on the Hydrogen Storage Capacity}

High pressure (0-3000 kPa) adsorption tests of hydrogen gas were carried out on a magnetic suspension balance (Rubotherm, Bochum, Germany). The balance contains an automatic gas sampling system, pressure control system, and temperature control system. In the experiment, the purge gas was high-purity helium (99.999\%), and the adsorption gas was high-purity hydrogen $(99.999 \%)$. First, the sample with the 1/3-1/2 sample frame volume was placed in a stainless steel sample box. Then, the pretreatment of samples was carried out in an electric oven at $150{ }^{\circ} \mathrm{C}$ under vacuum for $12 \mathrm{~h}$. Thereafter, the adsorption test was conducted with a controlled flow rate of $30 \mathrm{~mL} / \mathrm{min}$ for both the adsorption gas and the purge gas. The adsorption temperatures were set to $25{ }^{\circ} \mathrm{C}$ and $55^{\circ} \mathrm{C}$, and different measurement points were selected within the pressure range of 100-2500 $\mathrm{kPa}$. The adsorption instrument recorded the sample weights at each predefined pressure level. After data processing, the hydrogen adsorption isotherm under different temperatures can be obtained.

\section{Characterization of Graphite Oxide and Graphene Samples}

\subsection{XRD Characterization}

Figure 3 shows the XRD characteristics of graphite (G), graphite oxide (GO), and graphene (GS1/GS). In the figure, the crystal plane diffraction peak of graphite is extremely strong and sharp at $2 \theta=26.58^{\circ}$, indicating that the graphite sheets are laid neatly through the space. The graphite oxide crystal plane has a diffraction peak at $2 \theta=10.60^{\circ}$, with a relatively low intensity. This result suggests that the graphite interlayer spacing increases from $0.336 \mathrm{~nm}$ to $0.779 \mathrm{~nm}$ due to the oxidation process. When the graphite oxide was reduced to graphene, a diffraction peak appeared at $26.08^{\circ}$, and the diffraction peak became broader with decreased intensity. This can be explained by the decreased gap between the graphene sheets and higher disorder level. In Figure $3 b$, the diffraction peak of graphene GS1 is lower than that of GS, since the activity of dispersant hinders the agglomeration of graphene sheets. As the graphene GS1 that is prepared is mostly monolayered, the distance between layers is much larger than the wavelength of X-rays, which leads to inconspicuous diffraction. This demonstrates that adding dispersant is effective at creating monolayer graphene. 

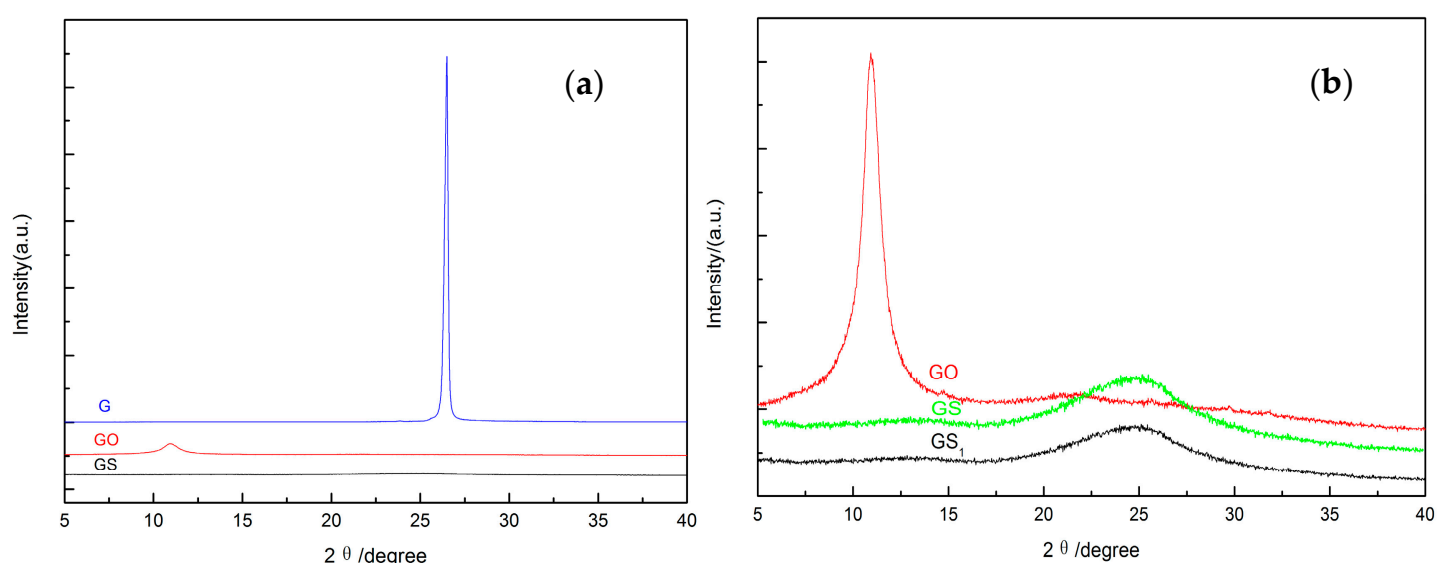

Figure 3. (a) X-ray diffraction (XRD) pattern of graphite (G), graphite oxide (GO), and graphene (GS); and (b) XRD magnification of GO, graphene (GS1), and GS.

\subsection{Raman Spectroscopy}

Figure 4 shows the Raman spectra of graphite (G), graphite oxide (GO), and graphene (GS1). The G sample has a sharp and strong absorption peak (G-peak) at $1576 \mathrm{~cm}^{-1}$ and a weak absorption peak (D-peak) at $1332 \mathrm{~cm}^{-1}$, indicating that the atomic structure sp2 hybrid carbon of graphite is very tight. After the graphite was oxidized, the G-peak of the graphite oxide becomes boarder and blue-shifts to $1578 \mathrm{~cm}^{-1}$, while the intensity of the D-peak increases. This result indicates that after oxidization, the structural symmetry decreased, the vibration mode increased, part of the sp2 hybrid carbon atoms were converted into sp3 hybrid structures, and $\mathrm{C}=\mathrm{C}$ double bonds were destroyed. In addition, the intensity ratio of the D-band to the G-band (ID/IG, sp3/sp2 carbon atomic ratio) suggests that the plane length of the sp2 hybridized carbon layer in graphite oxide is shorter than that of graphite. The ID/IG of graphite is 0.052 , which is obviously smaller than that of graphite oxide and graphene. Due to the supersonic effect, after the graphite was oxidized, the graphite layers were broken down, and the graphite layer area had shrunk considerably. After graphite oxide is reduced, the D-peak and G-peak were red-shifted. Its intensity ratio (ID/IG $=1.28$ ) is higher than that of the graphite oxide (ID/IG = 1.06), indicating that after the graphite has been fully oxidized and ultrasonically exfoliated, the average size of the $\mathrm{sp} 2$ hybridized carbon layers in graphene was larger than that of graphite oxide. At the same time, only a part of the sp3 hybrid carbon atoms were reduced to the sp2 hybrid carbon atom, that is, the structure of the graphite oxide cannot be completely restored to its original graphite structure.

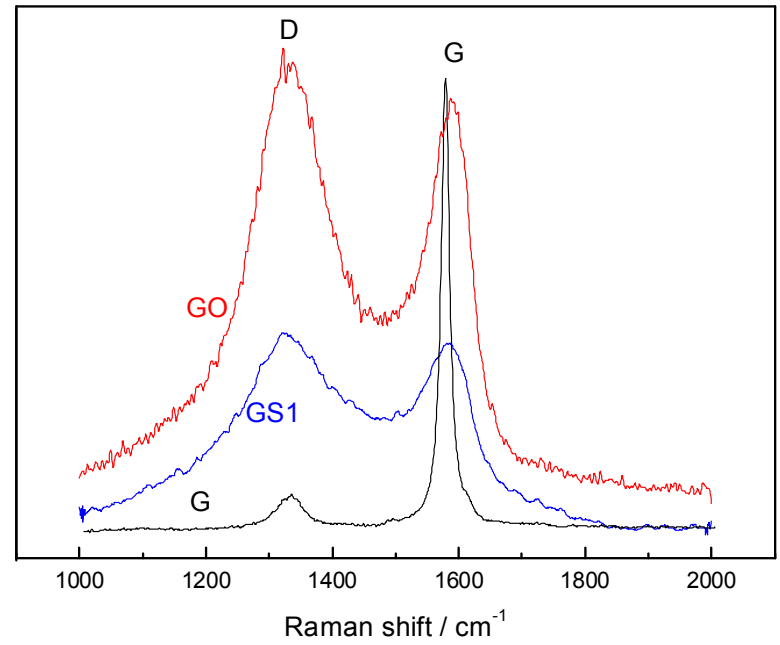

Figure 4. The Raman spectra of G, GO, and GS1. 


\subsection{FT-IR Characterization}

Figure 5 shows the infrared spectrum of graphite (G), graphite oxide (GO), and graphene (GS1). Before oxidization, due to the graphite six-membered ring having almost no other groups, the peak on the infrared spectrum is relative weak. After oxidization, peaks corresponding to $\mathrm{OH}\left(3414 \mathrm{~cm}^{-1}\right)$, $\mathrm{C}=\mathrm{O}\left(1723 \mathrm{~cm}^{-1}\right)$, aromatic ring $\mathrm{C}=\mathrm{C}\left(1627 \mathrm{~cm}^{-1}\right)$, and epoxy $\mathrm{CO}\left(1233 \mathrm{~cm}^{-1}\right)$ appeared on the infrared spectrum of graphite oxide. The $\mathrm{OH}$ vibration peak was generated due to the vibration of hydroxyl groups in the water molecules between the graphite layers. The carbonyl, carboxyl, and epoxy groups result from graphite oxidation. The carbonyl peak is very weak, as it can only be formed close to the graphite layer edge. The epoxy and the alkoxy groups exhibit strong peaks, since the graphite oxide contains a large amount of hydroxyl and epoxy groups. After the graphite oxide was reduced, the oxygen-containing functional group peak of the graphene sample significantly weakened or even disappeared, while the aromatic ring $C=C\left(1627 \mathrm{~cm}^{-1}\right)$ peak was enhanced. Most of the oxygen-containing functional groups in the graphene were removed, but the crystal structure is different from that of the original graphite.

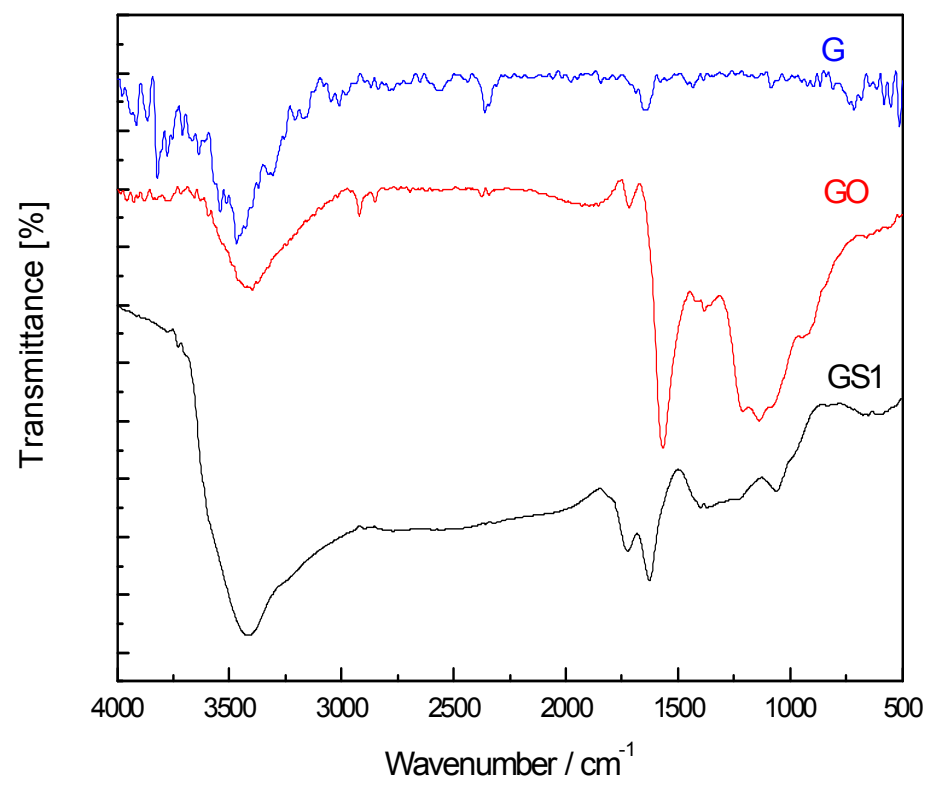

Figure 5. Fourier transform infrared (FT-IR) spectra for graphite, graphite oxide, and graphene.

\subsection{XPS Characterization}

Figure 6 shows the XPS spectra of graphite oxide and surfactant-modified graphene. The XPS test shows that the characteristic peaks, C1s and O1s, appear at $283 \mathrm{eV}$ and $534 \mathrm{eV}$, respectively. The carbon-to-oxygen atomic ratios of graphite oxide and graphene are 2.11 and 7.06, respectively, indicating that the graphite is fully oxidized. The XPS spectrum of graphite oxide in Figure 6a shows that the sp2 hybridized $\mathrm{C}-\mathrm{C}$ skeleton characteristic peak and $\mathrm{C}-\mathrm{O}-\mathrm{C}$ epoxy functional group peak appear at $285 \mathrm{eV}$ and $287 \mathrm{eV}$, respectively, while the weak carboxyl characteristic peak appears at $289 \mathrm{eV}$, suggesting the existence of oxygen-containing groups. After reduction, the characteristic peaks of the modified graphene, including epoxy and carboxyl groups, are significantly reduced. This result agrees with results of previous infrared tests $[35,36]$. 

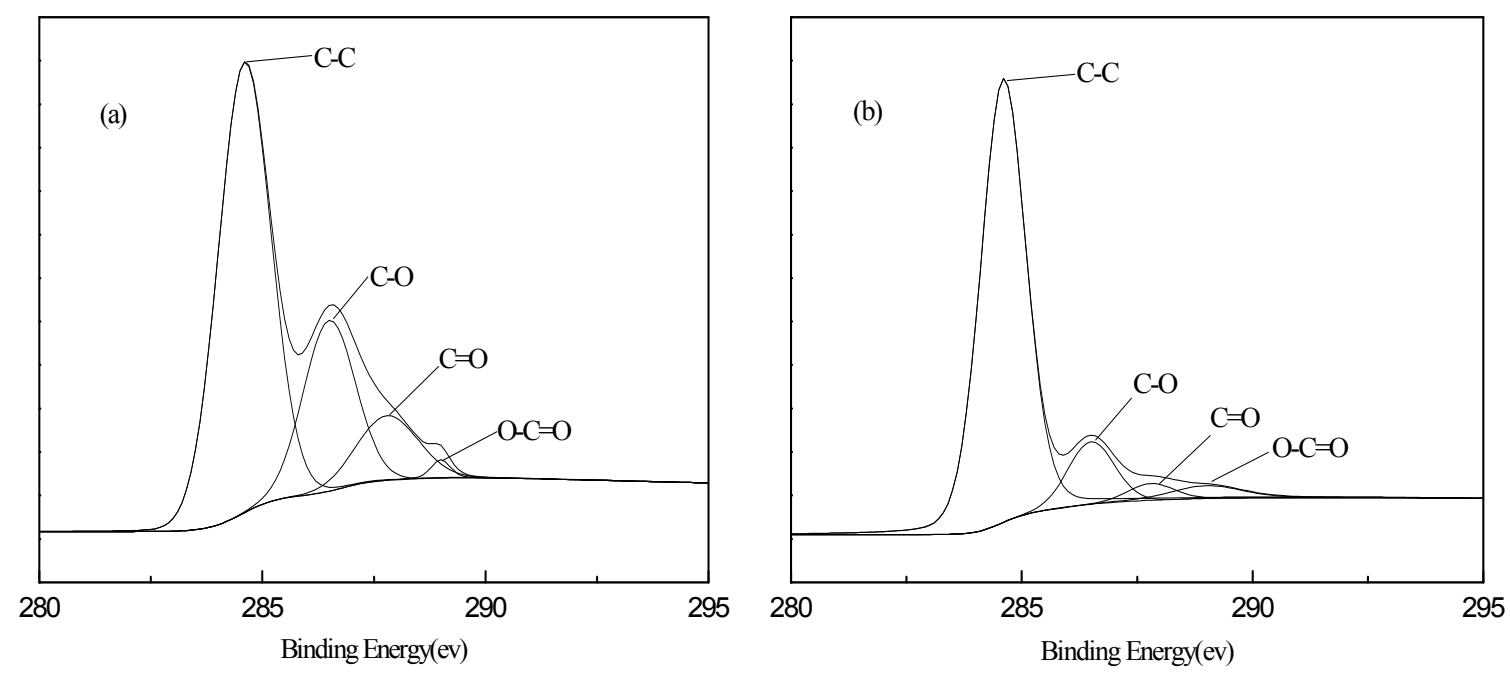

Figure 6. The XPS spectra of (a) graphite oxide and (b) graphene.

\subsection{TG Analysis}

Figure 7 shows the TG curves for GO and GS1. When the graphite oxide was heated to $800{ }^{\circ} \mathrm{C}$, the process has two obvious weight loss steps. The mass loss below $150{ }^{\circ} \mathrm{C}$ was mainly caused by the volatilization of the water molecules adsorbed by the graphite oxide, and the mass loss between $180-250^{\circ} \mathrm{C}$ may be due to the heat generation in the oxygen-containing groups. The decomposition process generates $\mathrm{CO}, \mathrm{CO}_{2}$, and $\mathrm{H}_{2} \mathrm{O}$. The GS1 had a slight mass loss below $160^{\circ} \mathrm{C}$ due to the small amount of adsorbed water molecules. Between $160-800^{\circ} \mathrm{C}$, the mass loss was significantly reduced compared to curve (a), indicating that the GS1 sample has better thermal stability.

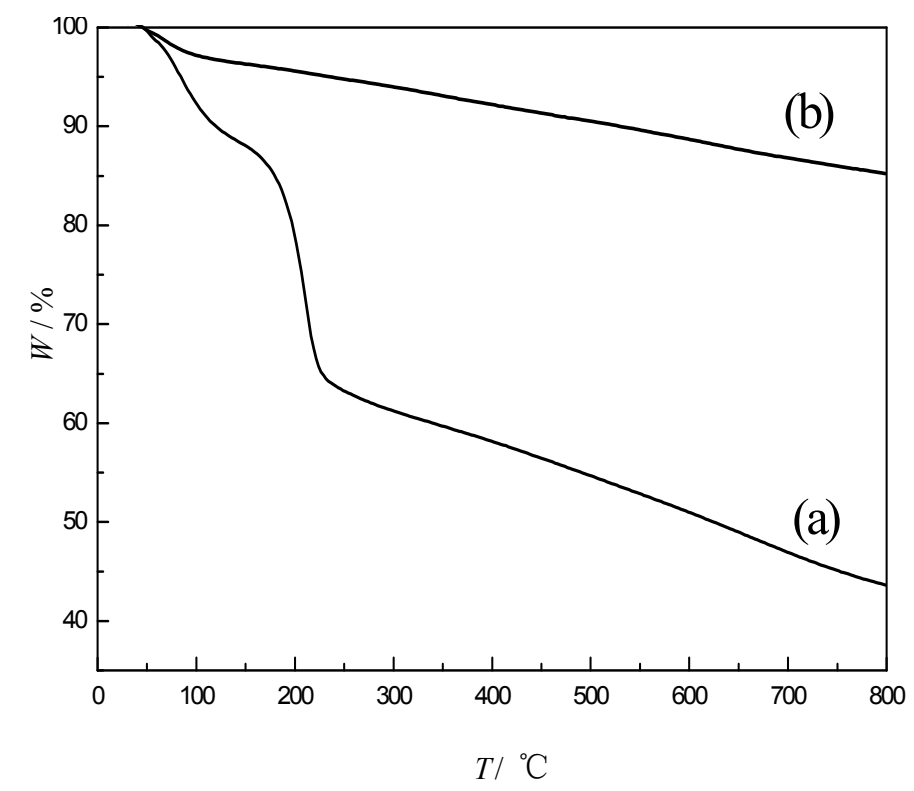

Figure 7. Thermogravimetric curves of (a) GO and (b) GS1.

\section{6. $\mathrm{N}_{2}$ Adsorption-Desorption Characterization}

Figure 8 show the $\mathrm{N}_{2}$ adsorption-desorption isotherms and pore size distributions of graphene samples GS1 and GS at liquid nitrogen temperature, respectively. From the figure, the isotherm shows a type I adsorption curve, and a hysteresis loop appears on the isothermal adsorption-desorption curve at a relative pressure of about 0.4 , which results in a narrow gap between graphene layers. The specific surface area of GS1 (about $\left.1206 \mathrm{~m}^{2} \cdot \mathrm{g}^{-1}\right)$ is much larger than that of GS $\left(605 \mathrm{~m}^{2} \cdot \mathrm{g}^{-1}\right)$, but smaller than 
the theoretical surface area of single-layer graphene $\left(2630 \mathrm{~m}^{2} \cdot \mathrm{g}^{-1}\right)$. This shows that the graphene sheet GS1 has both single-layer graphene and double-layered structures. However, compared with previous studies, the specific surface is still significantly improved [27]. The pore size distribution in Figure $8 \mathrm{~b}$ also shows that, compared with GS, GS1 has a smaller pore size and a larger number of micropores. This explains why the specific surface area of GS1 is larger than that of GS.
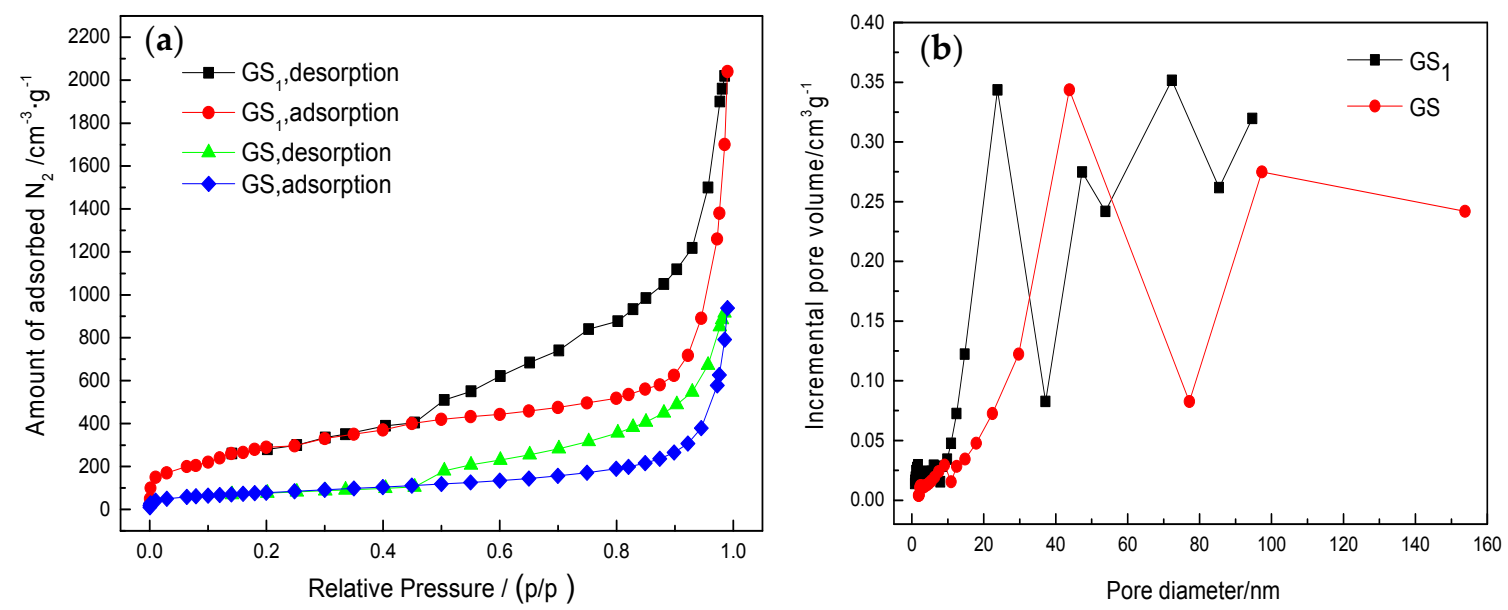

Figure 8. (a) $\mathrm{N}_{2}$ adsorption-desorption isotherms of GS1 and GS and (b) pore size distributions of GS1 and GS.

\subsection{Morphological Representation}

Figure 9 shows the SEM (a, b) and TEM (c, d) images of graphene GS1 and GS, respectively. In Figure 9a, the graphene GS1 is composed of a layer of honeycomb monolithic structures with a well-arranged and porous structure, indicating that there is very little agglomeration during the reduction process. In Figure 9b, graphene GS is stacked by layers, indicating significant agglomeration during the reduction process. Due to the dispersant, the dispersion of GS1 is significantly improved. In Figure 9c, the GS1 has an almost transparent, thin silk-like structure with a smooth, regular surface and fewer defects, while also containing fewer graphene layers. In Figure 9d, the surface of GS is uneven, and its thickness is also non-uniform, indicating that GS includes a large number of overlapped and curled single-layer graphene. The results show that adding dispersant can effectively improve the quality of graphene produced through the reduction of graphite oxide.

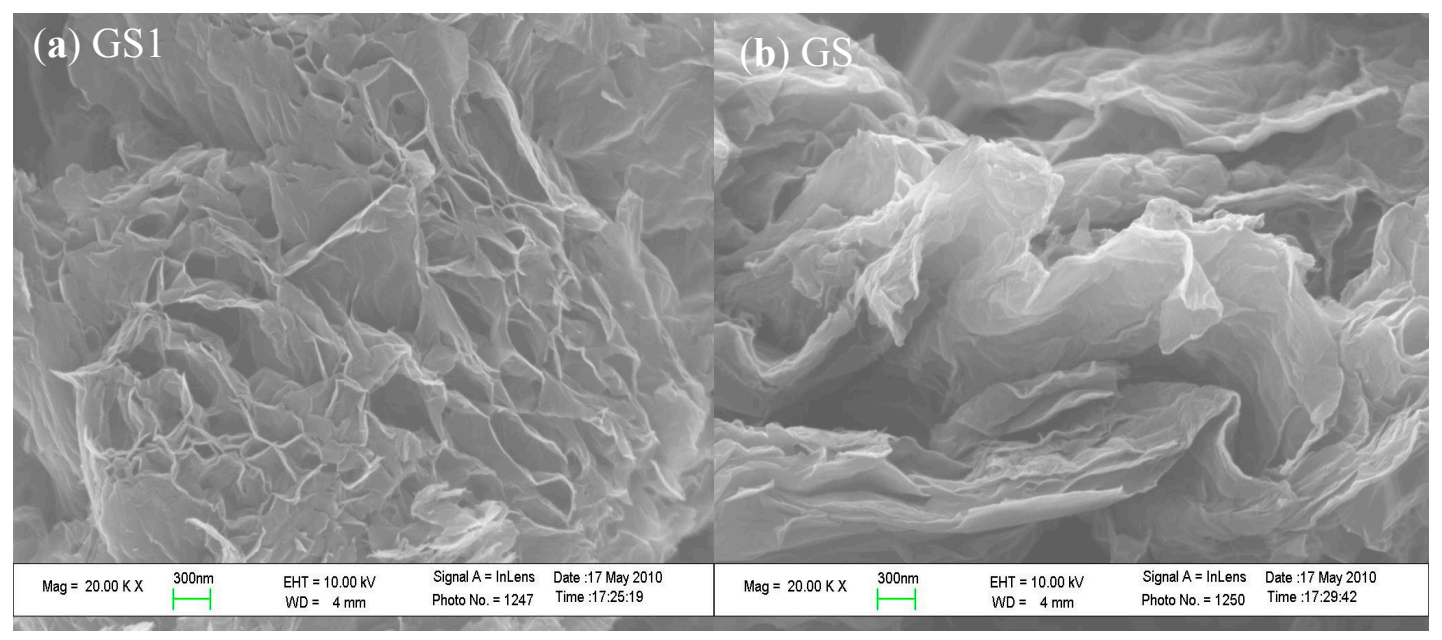

Figure 9. Cont. 


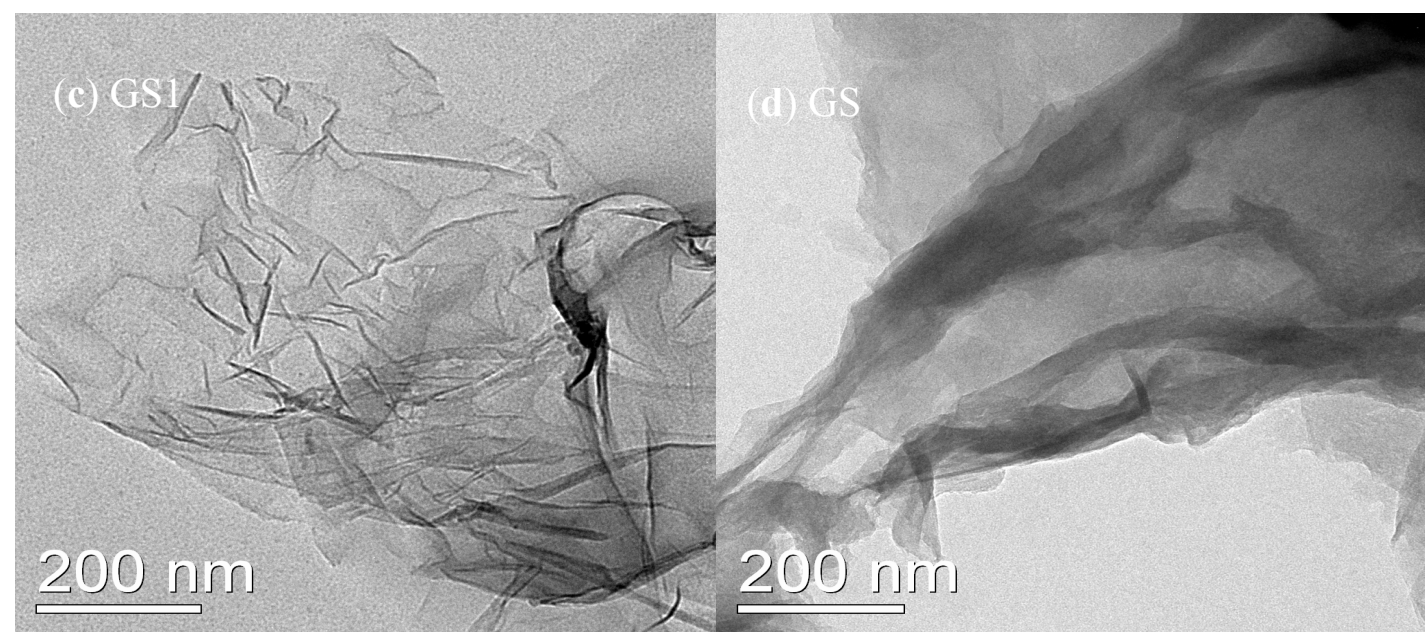

Figure 9. $(\mathbf{a}, \mathbf{b})$ SEM images and (c,d) TEM views of GS1 and GS.

Figure 10 displays an atomic force microscopy (AFM) image of the GS1. In the figure, the graphene nanosheets are evenly dispersed on a mica sheet substrate. The sample shows a stacked state with uniform colors that can be clearly distinguished. Stankovich et al. [37] have theoretically demonstrated and experimentally shown that the single-layer graphene produced by the liquid oxidation-reduction method has a thickness of about $1.1 \mathrm{~nm}$. According to the AFM analysis, the average thickness of GS1 is about $1.1 \mathrm{~nm}$, which suggests a single graphene layer, while very few double or triple graphene layers also exist in the sample.
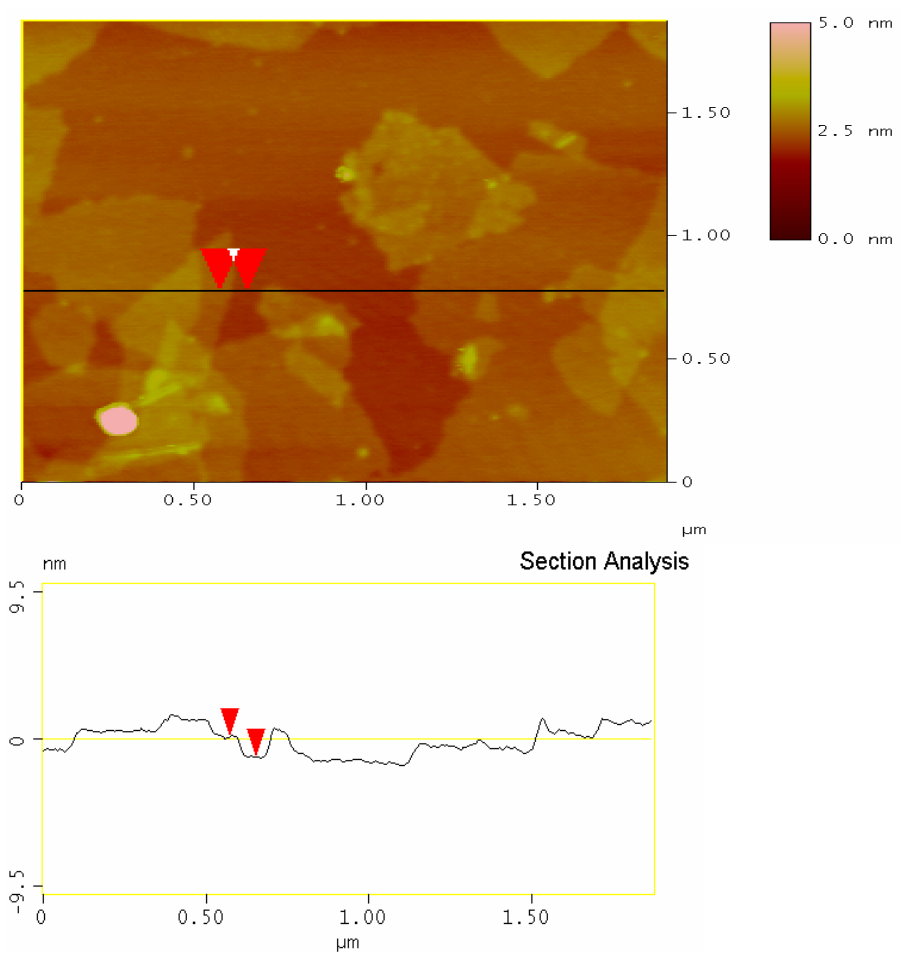

Figure 10. Atomic force microscopy (AFM) image of GS1.

\subsection{Dispersibility of Graphene Aqueous Solution}

Figure 11 compares the solubility, dispersion, and stability of graphene suspension (concentration $5 \mathrm{mg} / \mathrm{mL}$ ) before and after adding $100 \mathrm{~mL}$ of dispersant. It can be seen from the figure that most of the unmodified graphene sinks to the bottom, and obvious delamination and instability occur. The modified graphene remains stable in aqueous solution. The dispersant reduces the surface energy 
between the particles, which improves the hydrophilicity of the graphene. The modified graphene has a good water solubility and dispersibility, and remains stable for more than 60 days.

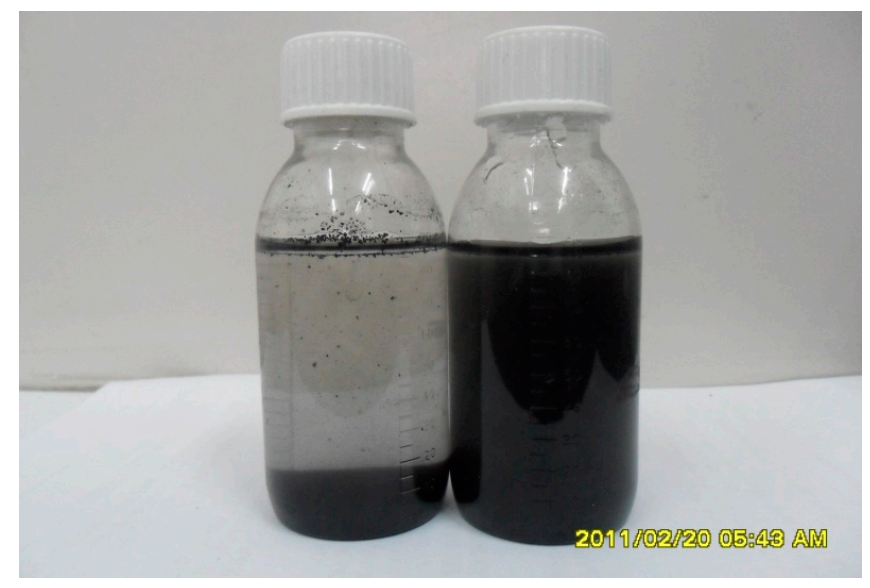

Figure 11. Comparison of graphene suspension before and after adding SDBS for 60 days.

\section{Validations of the Hydrogen Storage Capacity}

\subsection{Hydrogen Adsorption}

Figure 12 shows the comparison of experimental results of high-pressure hydrogen adsorption on GS1 and GS prepared under different pressure and temperature. In the figure, the adsorption capacity of graphene GS1 is significantly higher than that of GS. At $25^{\circ} \mathrm{C}$ and $55{ }^{\circ} \mathrm{C}$, the hydrogen adsorption of graphene GS1 and GS varies under different pressures. At the same temperature, with the increase of pressure, the adsorption amount on graphene gradually increases; at the same pressure level, the adsorption amount decreases with the increase of temperature.

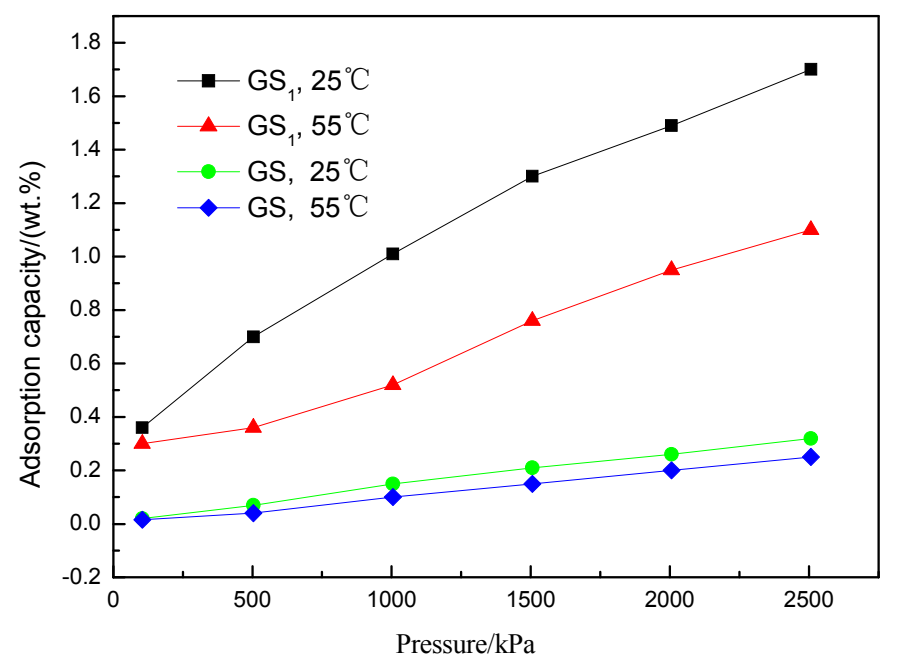

Figure 12. $\mathrm{H}_{2}$ adsorption capacity of GS1 and GS under different pressures at $25^{\circ} \mathrm{C}$ and $55^{\circ} \mathrm{C}$.

When the hydrogen pressure is relatively low, hydrogen molecules are mainly adsorbed in the micropores. As GS1 has more micropores, its adsorption capacity is better than GS in the early stages. With the increase of pressure, the hydrogen is mainly adsorbed by the mesopores and macrospores, and the adsorption amount increases dramatically. For example, at $25^{\circ} \mathrm{C}$ and $2500 \mathrm{kPa}$, the amount of hydrogen adsorbed by GS1 is as high as $1.7 \mathrm{wt} \%$. The heat of adsorption of hydrogen for graphene at temperatures $T_{1}$ and $T_{2}$ can be calculated by the Clausius-Clapeyron equation (as below, Equation (1)) [27], which is approximately $18.6 \mathrm{~kJ} / \mathrm{mol}$. This value is close to the adsorption heat $(15 \mathrm{~kJ} / \mathrm{mol})$ of the ideal adsorbent material suggested by the United States Department of Energy [38]. 
Clausius-Clapeyron equation:

$$
Q_{s t}=\frac{R \cdot \ln \left(P_{T_{1}} / P_{T_{2}}\right)}{1 / T_{1}-1 / T_{2}}
$$

where $Q_{s t}$ is the heat of adsorption $(\mathrm{kJ} / \mathrm{mol}), R$ is the gas constant $(8.314 \mathrm{~kJ} /(\mathrm{mol} \mathrm{K}))$, and $P_{T 1}$ and $P_{T 2}$ are the hydrogen pressures $(\mathrm{kPa})$ at temperatures $T_{1}$ and $T_{2}$, respectively.

\subsection{Hydrogen Storage Capacity-Fitting}

In order to analyze the rule of adsorption properties of graphene, two kinds of hydrogen adsorption models are discussed.

\subsubsection{Langmuir Model}

Langmuir isothermal adsorption mode (Equation (2)) is the first model to describe the adsorption mechanism vividly, which lays a foundation for the establishment of other adsorption models. The model assumes that the adsorbent surface is uniform and there is no interaction between the adsorbents. Also, the adsorption is considered as only occuring on the external surface of the adsorbent.

$$
\frac{p}{V^{a}}=\frac{1}{V_{m}^{a} \cdot b}+\frac{p}{V_{m}^{a}}
$$

where $V_{m}^{a}$ is the gas volume during adsorption saturation, $V^{a}$ is the gas volume during adsorption equilibrium, $b$ is the adsorption equilibrium constant, which is a dimensionless parameter, and $V_{m}^{a}$ and $b$ can be estimated with regression slope and intercept, respectively.

\subsubsection{Freundlich Adsorption Model}

Freundlich isothermal adsorption model (Equation (3)) can be applied to both single-layer adsorption and non-uniform surface adsorption, which can describe the adsorption mechanism of non-uniform surface and is more suitable for low concentration adsorption.

$$
\lg V^{a}=\lg K+n \cdot \lg p
$$

where $V^{a}$ is the actual amount of adsorption $(\mathrm{mL} / \mathrm{mg})$ under pressure, $K$ is the reaction rate constant, $n$ is the empirical constant, and $p$ is the adsorption pressure. A smaller $n$ means a better adsorption ability. In general, when $n$ is between $0.1-0.5$, the adsorption is easy to perform; when $n>2$, adsorption is more difficult. $V^{a}$ can be measured by the experimental method, and then $K$ and $n$ can be calculated through the above model. Figure 13 shows the results of both fitting models.

(a)

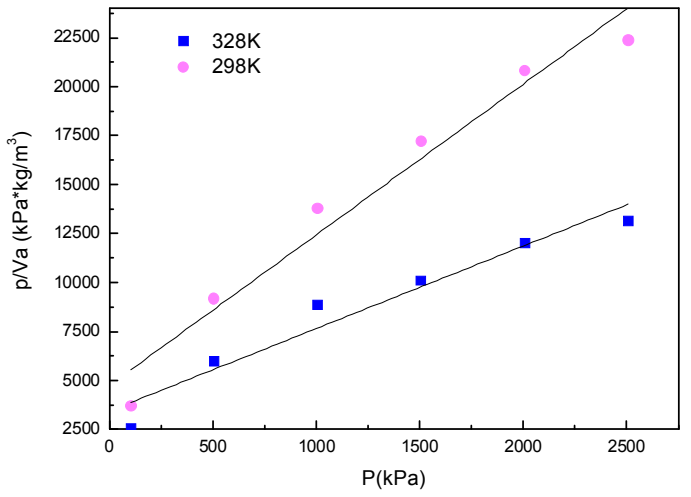

(b)

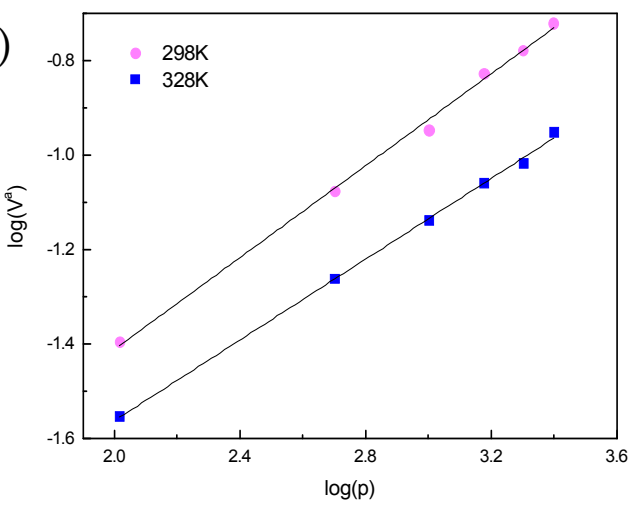

Figure 13. (a) Hydrogen adsorption capacity of graphene with the Langmuir model and (b) Freundlich model.

The adsorption isotherms of hydrogen on graphene samples were fitted with Langmuir adsorption isotherm equations and Freundlich adsorption isotherm equations, as shown in Figure 13. The fitting functions of the Langmuir adsorption isotherm equation and the Freundlich adsorption isotherm 
equation agree with the experiment results. The Freundlich adsorption isotherm equation shows a better fitting result. Table 3 lists the fitting parameters and variances for the adsorption isotherms. The correlation coefficients of both the Langmuir adsorption isotherm equation and the Freundlich adsorption isotherm equation are above 0.94 .

Table 3. Fitting parameters of Langmuir and Freundlich adsorption isotherm equations.

\begin{tabular}{ccccccc}
\hline \multirow{2}{*}{$\boldsymbol{T}(\mathbf{K})$} & \multicolumn{3}{c}{ Langmuir Equation } & \multicolumn{4}{c}{ Freundlich Equation } \\
\cline { 2 - 7 } & $\mathbf{b}$ (mg/g) & $\boldsymbol{V}_{\boldsymbol{m}}^{\boldsymbol{a}}$ (mL/mg) & $\mathbf{R}^{\mathbf{2}}$ & $\mathbf{K}$ & $\mathbf{n}$ & $\mathbf{R}^{\mathbf{2}}$ \\
\hline 298 & 0.00123 & 0.2374 & 0.946 & 0.00414 & 0.486 & 0.997 \\
328 & 0.00162 & 0.1300 & 0.963 & 0.00384 & 0.427 & 0.999 \\
\hline
\end{tabular}

Form Table 3, at $298 \mathrm{~K}$ and $328 \mathrm{~K}$, the correlation coefficient of the Freundlich adsorption isotherm equation is better than that of the Langmuir adsorption isotherm equation. The Freundlich adsorption isotherm equation was used to describe the adsorption isotherms, and it illustrates the existence of the peripheral curl and stacked states of the graphene sample, which is non-monolayer adsorption. The Langmuir adsorption model emphasizes the interaction between the monolayer and the adsorption medium, while the Freundlich adsorption model is a more flexible semi-empirical equation. The Freundlich adsorption model also shows that the adsorption of hydrogen molecules on graphene is a physical process. When hydrogen is adsorbed on graphene at low pressure, it first occurs in the micropores and hollow structure of graphene. With the increase of equilibrium pressure, the adsorption of hydrogen molecules gradually occurs on the outer surface of graphene, which is in line with the physical adsorption characteristics.

\section{Conclusions}

This paper proposed preparing graphite oxide through a liquid phase oxidation method. After ultrasonic peeling, a high-quality graphene sheet can be prepared by adding the dispersant sodium dodecyl benzene sulfonate (SDBS) during the reduction process. The structure, morphology and composition of the prepared samples were inspected by XRD, Raman, Fourier transform infrared (FT-IR), TG, SEM, TEM, and AFM. The hydrogen adsorption properties of graphene then were investigated by conducting hydrogen adsorption experiments. Through characterization analysis and experimental results, this study concludes:

(1) Adding the dispersant sodium dodecylbenzene sulfonate can greatly improve the quality of the prepared graphene sheets. The final prepared product is mainly composed of single-layer graphene with a thickness of $1.1 \mathrm{~nm}$ and a small amount of double-layer graphene.

(2) The $\mathrm{N}_{2}$ adsorption-desorption experiments show that the graphene sheet involves a large specific surface area with a rich pore structure. The application of dispersant sodium dodecyl benzene sulfonate (SDBS) can effectively reduce the agglomeration among graphene monolayers and increase the increase the specific surface area of graphene (from unmodified graphene $605 \mathrm{~m}^{2} \cdot \mathrm{g}^{-1}$ to improved $\left.1206 \mathrm{~m}^{2} \cdot \mathrm{g}^{-1}\right)$.

(3) In the hydrogen adsorption experiments, the adsorption capacity of high-quality graphene at $25^{\circ} \mathrm{C}$ and $55^{\circ} \mathrm{C}$ with pressure of $2500 \mathrm{kPa}$ reached $1.7 \mathrm{wt} \%$ and $1.1 \mathrm{wt} \%$, respectively. The adsorption isotherms of hydrogen on graphene samples were fitted by the Langmuir adsorption isotherm equation and the Freundlich adsorption isotherm equation. The fitting results show that the linearity of the Freundlich adsorption isotherm equation performs better.

(4) The Freundlich fitting model also shows that when hydrogen is adsorbed on graphene at low pressure, it first occurs in the micropore and hollow structures of graphene. With the increase of adsorption pressure, the adsorption of hydrogen molecules gradually occurs on the outer surface of graphene. 
Author Contributions: Conceptualization, W.Y.; formal analysis, B.L. and T.X.; investigation, B.L.; data curation, L.L.; writing—original draft preparation, T.X.; writing—review and editing, J.C.; visualization, H.W.; supervision, X.Z.

Funding: This research received no external funding.

Acknowledgments: This work was supported by Guangzhou Science and Technology Program (201704030137), the Research Project of Guangdong Province (2017A050506058), and China Fundamental Research Funds for the Central Universities (16lgpy20).

Conflicts of Interest: The authors declare no conflict of interest.

\section{References}

1. Haryanto, A.; Fernando, S.; Murali, N.; Adhikari, S. Current Status of Hydrogen Production Techniques by Steam Reforming of Ethanol: A Review. Energy Fuels 2005, 19, 2098-2106. [CrossRef]

2. Barelli, L.; Bidini, G.; Gallorini, F.; Servili, S. Hydrogen production through sorption-enhanced steam methane reforming and membrane technology: A review. Energy 2008, 33, 554-570. [CrossRef]

3. Rosen, M.A. Advances in hydrogen production by thermochemical water decomposition: A review. Energy 2010, 35, 1068-1076. [CrossRef]

4. Yang, J.; Wu, H.; Huang, G.; Liang, Y.; Liao, Y. Modeling and coupling effect evaluation of thermal conductivity of ternary opacifier/fiber/aerogel composites for super-thermal insulation. Mater. Des. 2017, 133, 224-236. [CrossRef]

5. Yang, J.; Wu, H.; Wang, M.; Liang, Y. Prediction and optimization of radiative thermal properties of nano $\mathrm{TiO}_{2}$ assembled fibrous insulations. Int. J. Heat Mass Transf. 2018, 117, 729-739. [CrossRef]

6. Khosravi, A.; Koury, R.N.N.; Machado, L.; Pabon, J.J.G. Energy, exergy and economic analysis of a hybrid renewable energy with hydrogen storage system. Energy 2018, 148, 1087-1102. [CrossRef]

7. Nagpal, M.; Kakkar, R. An evolving energy solution: Intermediate hydrogen storage. Int. J. Hydrog. Energy 2018, 43, 12168-12188. [CrossRef]

8. Assaf, J.; Shabani, B. Experimental study of a novel hybrid solar-thermal/PV-hydrogen system: Towards $100 \%$ renewable heat and power supply to standalone applications. Energy 2018, 157, 862-876. [CrossRef]

9. Khiareddine, A.; Ben Salah, C.; Rekioua, D.; Mimouni, M.F. Sizing methodology for hybrid photovoltaic/ wind/hydrogen/battery integrated to energy management strategy for pumping system. Energy 2018, 153, 743-762. [CrossRef]

10. Campíñez-Romero, S.; Colmenar-Santos, A.; Pérez-Molina, C.; Mur-Pérez, F. A hydrogen refuelling stations infrastructure deployment for cities supported on fuel cell taxi roll-out. Energy 2018, 148, 1018-1031. [CrossRef]

11. Onishi, N.; Laurenczy, G.; Beller, M.; Himeda, Y. Recent progress for reversible homogeneous catalytic hydrogen storage in formic acid and in methanol. Coord. Chem. Rev. 2018, 373, 317-332. [CrossRef]

12. Muir, S.S.; Yao, X. Progress in sodium borohydride as a hydrogen storage material: Development of hydrolysis catalysts and reaction systems. Int. J. Hydrog. Energy 2011, 36, 5983-5997. [CrossRef]

13. Yanik, M.O.; Yigit, E.A.; Akansu, Y.E.; Sahmetlioglu, E. Magnetic conductive polymer-graphene nanocomposites based supercapacitors for energy storage. Energy 2017, 138, 883-889. [CrossRef]

14. Züttel, A. Materials for hydrogen storage. Mater. Today 2003, 6, 24-33. [CrossRef]

15. Dillon, A.C.; Jones, K.M.; Bekkedahl, T.A.; Kiang, C.H.; Bethune, D.S.; Heben, M.J. Storage of hydrogen in single-walled carbon nanotubes. Nature 1997, 386, 377. [CrossRef]

16. Liu, C.; Fan, Y.Y.; Liu, M.; Cong, H.T.; Cheng, H.M.; Dresselhaus, M.S. Hydrogen Storage in Single-Walled Carbon Nanotubes at Room Temperature. Science 1999, 286, 1127-1129. [CrossRef] [PubMed]

17. Zhou, L.; Zhou, Y.; Sun, Y. A comparative study of hydrogen adsorption on superactivated carbon versus carbon nanotubes. Int. J. Hydrog. Energy 2004, 29, 475-479. [CrossRef]

18. Fan, Y.-Y.; Liao, B.; Liu, M.; Wei, Y.-L.; Lu, M.-Q.; Cheng, H.-M. Hydrogen uptake in vapor-grown carbon nanofibers. Carbon N. Y. 1999, 37, 1649-1652. [CrossRef]

19. Gupta, B.K.; Srivastava, O.N. Further studies on microstructural characterization and hydrogenation behaviour of graphitic nanofibres. Int. J. Hydrog. Energy 2001, 26, 857-862. [CrossRef]

20. Zhou, L.; Zhou, Y.; Sun, Y. Enhanced storage of hydrogen at the temperature of liquid nitrogen. Int. J. Hydrog. Energy 2004, 29, 319-322. [CrossRef] 
21. Novoselov, K.S.; Geim, A.K.; Morozov, S.V.; Jiang, D.; Zhang, Y.; Dubonos, S.V.; Grigorieva, I.V.; Firsov, A.A. Electric Field Effect in Atomically Thin Carbon Films. Science 2004, 306, 666-669. [CrossRef] [PubMed]

22. Choudhary, A.; Malakkal, L.; Siripurapu, R.K.; Szpunar, B.; Szpunar, J. First principles calculations of hydrogen storage on $\mathrm{Cu}$ and Pd-decorated graphene. Int. J. Hydrog. Energy 2016, 41, 17652-17656. [CrossRef]

23. Faye, O.; Eduok, U.; Szpunar, J.; Szpunar, B.; Samoura, A.; Beye, A. Hydrogen storage on bare Cu atom and Cu-functionalized boron-doped graphene: A first principles study. Int. J. Hydrog. Energy 2017, 42, 4233-4243. [CrossRef]

24. Luo, Z.; Fan, X.; Pan, R.; An, Y. A first-principles study of Sc-decorated graphene with pyridinic-N defects for hydrogen storage. Int. J. Hydrog. Energy 2017, 42, 3106-3113. [CrossRef]

25. Srinivas, G.; Zhu, Y.; Piner, R.; Skipper, N.; Ellerby, M.; Ruoff, R. Synthesis of graphene-like nanosheets and their hydrogen adsorption capacity. Carbon N. Y. 2010, 48, 630-635. [CrossRef]

26. Ghosh, A.; Subrahmanyam, K.S.; Krishna, K.S.; Datta, S.; Govindaraj, A.; Pati, S.K.; Rao, C.N.R. Uptake of $\mathrm{H}_{2}$ and $\mathrm{CO}_{2}$ by Graphene. J. Phys. Chem. C 2008, 112, 15704-15707. [CrossRef]

27. Ma, L.-P.; Wu, Z.-S.; Li, J.; Wu, E.-D.; Ren, W.-C.; Cheng, H.-M. Hydrogen adsorption behavior of graphene above critical temperature. Int. J. Hydrog. Energy 2009, 34, 2329-2332. [CrossRef]

28. Bourlinos, A.B.; Gournis, D.; Petridis, D.; Szabó, T.; Szeri, A.; Dékány, I. Graphite Oxide: Chemical Reduction to Graphite and Surface Modification with Primary Aliphatic Amines and Amino Acids. Langmuir 2003, 19, 6050-6055. [CrossRef]

29. Niyogi, S.; Bekyarova, E.; Itkis, M.E.; McWilliams, J.L.; Hamon, M.A.; Haddon, R.C. Solution Properties of Graphite and Graphene. J. Am. Chem. Soc. 2006, 128, 7720-7721. [CrossRef] [PubMed]

30. Ensafi, A.A.; Jafari-Asl, M.; Nabiyan, A.; Rezaei, B.; Dinari, M. Hydrogen storage in hybrid of layered double hydroxides/reduced graphene oxide using spillover mechanism. Energy 2016, 99, 103-114. [CrossRef]

31. Zhao, G.; Li, J.; Wang, X. Kinetic and thermodynamic study of 1-naphthol adsorption from aqueous solution to sulfonated graphene nanosheets. Chem. Eng. J. 2011, 173, 185-190. [CrossRef]

32. Liang, Y.; Wu, D.; Feng, X.; Müllen, K. Dispersion of Graphene Sheets in Organic Solvent Supported by Ionic Interactions. Adv. Mater. 2009, 21, 1679-1683. [CrossRef]

33. Lu, X.; Xiao, Y.; Lei, Z.; Chen, J.; Zhang, H.; Ni, Y.; Zhang, Q. A promising electrochemical biosensing platform based on graphitized ordered mesoporous carbon. J. Mater. Chem. 2009, 19, 4707-4714. [CrossRef]

34. Lin, Y.S.; Haynes, C.L. Synthesis and Characterization of Biocompatible and Size-Tunable Multifunctional Porous Silica Nanoparticles. Chem. Mater. 2009, 21, 3979-3986. [CrossRef]

35. Pfaffeneder-Kmen, M.; Casas, I.F.; Naghilou, A.; Trettenhahn, G.; Kautek, W. A Multivariate Curve Resolution evaluation of an in-situ ATR-FTIR spectroscopy investigation of the electrochemical reduction of graphene oxide. Electrochim. Acta 2017, 255, 160-167. [CrossRef]

36. Pei, S.; Cheng, H.-M. The reduction of graphene oxide. Carbon N. Y. 2012, 50, 3210-3228. [CrossRef]

37. Stankovich, S.; Dikin, D.A.; Piner, R.D.; Kohlhaas, K.A.; Kleinhammes, A.; Jia, Y.; Wu, Y.; Nguyen, S.T.; Ruoff, R.S. Synthesis of graphene-based nanosheets via chemical reduction of exfoliated graphite oxide. Carbon N. Y. 2007, 45, 1558-1565. [CrossRef]

38. Jhi, S.H.; Kwon, Y.K.; Bradley, K.; Gabrief, J.C.P. Hydrogen storage by physisorption: Beyond carbon. Solid State Commun. 2004, 129, 769-773. [CrossRef]

(C) 2018 by the authors. Licensee MDPI, Basel, Switzerland. This article is an open access article distributed under the terms and conditions of the Creative Commons Attribution (CC BY) license (http://creativecommons.org/licenses/by/4.0/). 